\title{
The use of modern interventional cardiology tools to verify lesion significance and optimize procedural outcome in a diabetic patient with multivessel disease
}

\author{
Ziad Dahdouh ${ }^{1}$, Bahaa M. Fadel ${ }^{1}$, Hani Al Sergani ${ }^{1}$, Jehad Al Buraiki ${ }^{1}$, Abdalkareem Al Allaf ${ }^{1}$, Aysha Husain ${ }^{1}$, \\ Wafa Aldawood ${ }^{1}$, Vincent Roule ${ }^{2}$, Gilles Grollier ${ }^{2}$ \\ ${ }^{1}$ King Faisal Specialist Hospital and Research Center, Riyadh, Saudi Arabia \\ ${ }^{2}$ Department of Interventional Cardiology, University Hospital of Caen, Caen, France
}

Postep Kardiol Inter 2015; 11, 3 (41): 233-238

DOI: $10.5114 /$ pwki.2015.54024

\section{Introduction}

Coronary artery bypass graft (CABG) surgery has been the preferred revascularization technique for multivessel coronary artery disease, particularly in patients with diabetes mellitus [1-4]. However, with the rapid progress in the field of interventional cardiology, percutaneous coronary intervention $(\mathrm{PCI})$ has been widely used as a less invasive approach to CABG surgery, with encouraging results. Here we present the case of a patient with multivessel coronary artery disease who was managed by $\mathrm{PCl}$ using modern interventional tools to improve on diagnostic angiographic data and optimize the procedural result.

\section{Case report}

A 52-year-old man with a history of cigarette smoking, diabetes mellitus and old inferolateral myocardial infarction was referred to our institution for CABG surgery. Following the recent onset of epigastric pain and dyspnea, he underwent cardiac work-up. A resting electrocardiogram was normal. An echocardiogram demonstrated a mildly reduced left ventricular ejection fraction (LVEF) at 45\% with hypokinesis of the inferolateral wall. A coronary angiogram done at the local hospital reportedly showed evidence of severe 3-vessel disease.

On review of the coronary angiogram at our institution, the dominant right coronary artery (RCA) demonstrated a hazy lesion suggestive of a ruptured plaque in its proximal segment along with severe stenosis in its third segment (95\%). The left circumflex (LCX) artery showed severe narrowing in its proximal (80\%) and mid segments (99\%), the latter at the take-off of the first ob- tuse marginal (OM1) branch, which also demonstrated severe ostial disease (85\%). The left anterior descending (LAD) artery exhibited long lesions in the proximal and mid segments, visually in the $60-70 \%$ stenosis range (Figure 1 A). The logistic Syntax score was calculated at $29 \%$. The cumulative 2-year major adverse cardiac and cerebrovascular event (MACCE) rate is $16.4 \%$ by CABG and $22.8 \%$ by $\mathrm{PCl}$. A fractional flow rate (FFR) study was done through the right radial approach to assess the hemodynamic significance of the LAD lesions (Figure 1 B). An FFR of 0.85 following hyperemia induced by intravenous adenosine through the left brachial vein confirmed the lack of physiological influence on coronary flow. Therefore a clinical decision was made favoring a staged percutaneous revascularization over CABG. An intravascular ultrasound (IVUS) study was then performed on the proximal RCA lesion (Figure $1 \mathrm{C}$ ). It demonstrated the presence of a coronary dissection at the site with a false and true lumen separated by an intimal flap (Figure $1 \mathrm{D}$ ). Following pre-dilatation of the mid RCA lesion using a $2 \mathrm{~mm} \times 12 \mathrm{~mm}$ Mini Trek balloon (Abbott Vascular, Santa Clara, CA, USA), three overlapping drug-eluting stents (DES) $(3 \mathrm{~mm} \times 22 \mathrm{~mm}, 3.5 \mathrm{~mm}$ $\times 18 \mathrm{~mm}$ and $3.5 \mathrm{~mm} \times 30 \mathrm{~mm}$; Resolute Integrity, Medtronic, Inc., Santa Rosa, CA, USA) were deployed, extending from the mid to the ostial RCA (Figure $1 \mathrm{E}$ ) with a good angiographic result (Figure $1 \mathrm{~F}$ ). Post dilatation to the very proximal RCA was carried out using a $4 \mathrm{~mm} \times 12 \mathrm{~mm}$ non-compliant Trek NC balloon inflated to $15 \mathrm{~atm}$. Post stenting IVUS demonstrated good strut apposition to the vessel wall (Figure $1 \mathrm{G}, \mathrm{H}$ ). One month later, $\mathrm{PCl}$ of the LCx (Figure $2 \mathrm{~A}$ ) was performed. Using a 6 Fr Runway guiding catheter CLS 3 (Boston Scientific,

\section{Corresponding author:}

Ziad Dahdouh MD, King Faisal Specialist Hospital and Research Center, Riyadh, Saudi Arabia, fax: +33629362232,

e-mail: ziad_dahdouh@hotmail.com

Received: 21.11.2014, accepted: 11.03.2015. 

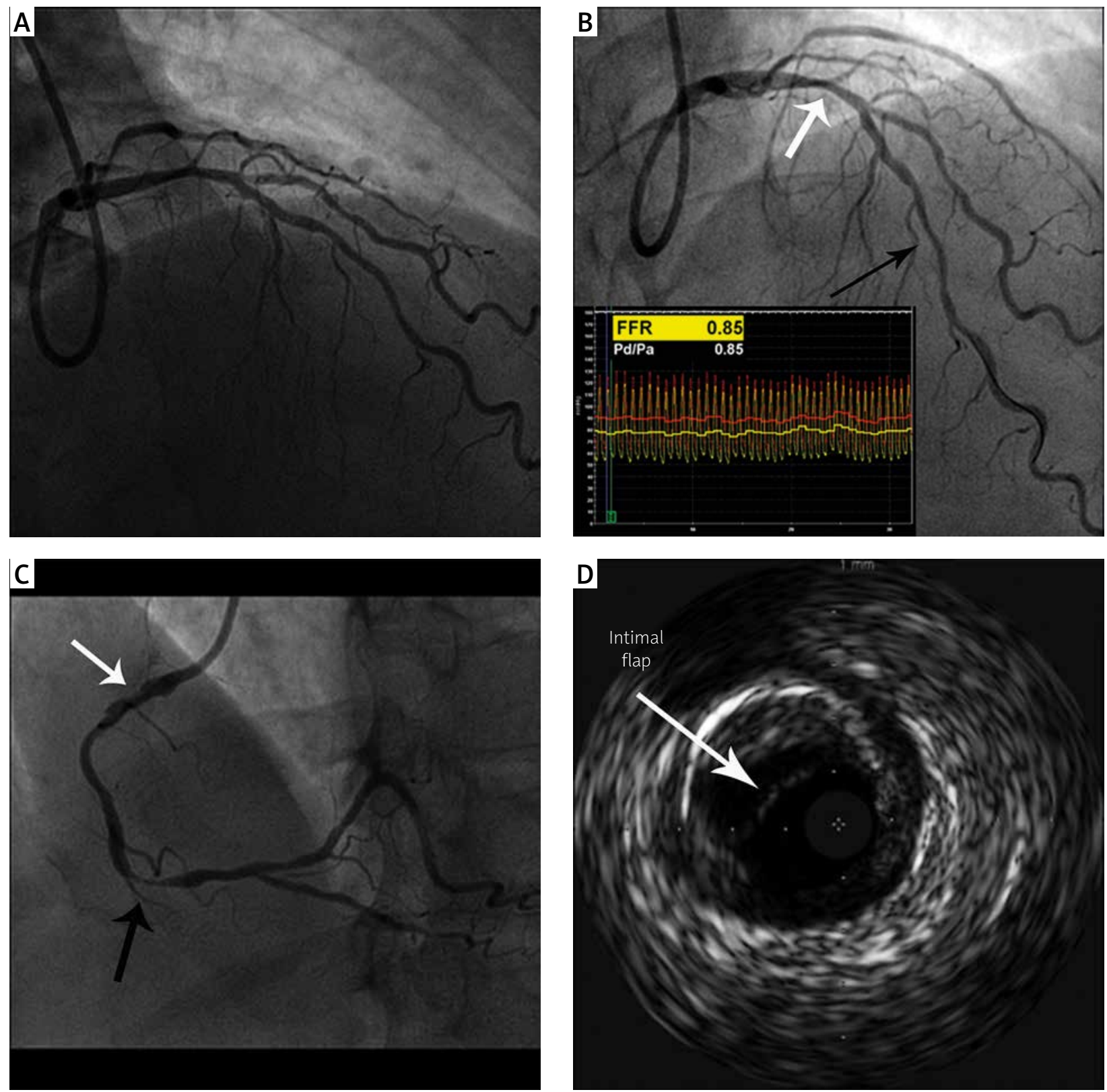

Figure 1. A - Angiography of the left anterior descending artery (LAD) showing long stenosis of the proximal and mid part. B - Fractional flow rate (FFR) guidewire and pressure curves to assess the hemodynamic significance of the LAD lesions (white arrow to the proximal segment and black arrow to the distal segment). C - Angiography of the right coronary artery (RCA) showing a hazy lesion (white arrow) in the proximal segment along with a severe stenosis in the third segment (black arrow). D - Intra-vascular ultrasound (IVUS) study in the proximal RCA showing an aspect of coronary dissection at the site with a false and true lumen separated by an intimal flap (white arrow)

Natick, MA, USA), two 0.014-in BMW guidewires were advanced, one to the distal LCX and the other to OM1. After pre-dilatation of the proximal and mid LCx using a $2.0 \mathrm{~mm} \times 20 \mathrm{~mm}$ Pantera balloon (Biotronik, Berlin, Germany), a $2.0 \mathrm{~mm} \times 15 \mathrm{~mm}$ Pantera Drug-coated Balloon was inflated in the mid LCx distal to OM1 up to 7 atmospheres (Figure $2 \mathrm{~B}$ ). A $2.5 \mathrm{~mm} \times 20 \mathrm{~mm}$ Trek balloon was then inflated in the proximal $L C X$ to OM1 at $10 \mathrm{~atm}$ and a DES $(2.75 \mathrm{~mm} \times 30 \mathrm{~mm}$; Resolute Integrity) was deployed (Figure $2 \mathrm{C}$ ), followed by post-dilatation using a $3.0 \mathrm{~mm} \times 12 \mathrm{~mm} \mathrm{NC}$ Trek balloon at $15 \mathrm{~atm}$ with a good angiographic result (Figure 2 D). Post-stenting optical coherence tomography (OCT) was performed and showed good strut apposition to the vessel wall (Figure $2 \mathrm{E}, \mathrm{F}$ ). The patient remained symptom-free at 6 months of follow-up. 

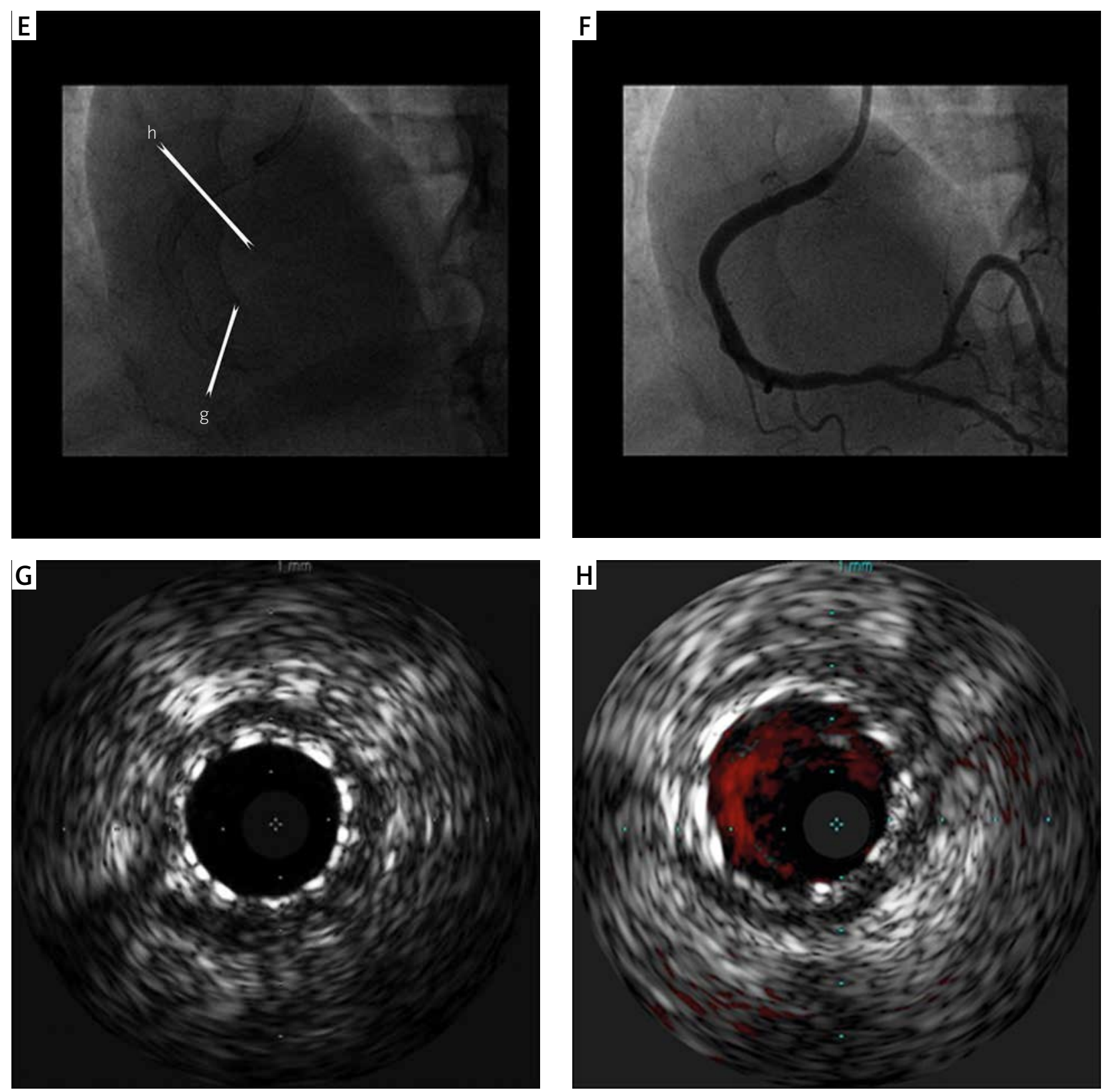

Figure 1. E, F - Fluoroscopy and angiography showing the three overlapping drug-eluting stents (DES) deployed from the third segment $(\mathrm{g})$ to the ostial (h) RCA with a good angiographic result with no residual stenosis or dissection. G, H - Post-stenting intra-vascular ultrasound (IVUS) study showing good strut apposition to the vessel wall in the third segment $(\mathrm{G})$ and proximal RCA $(\mathrm{H})$

\section{Discussion}

Management of coronary artery disease has been the subject of clinical trials aiming to provide specific recommendations for the preferred strategy of revascularization. Randomized clinical trials have favored CABG over $\mathrm{PCl}$ for patients with 3-vessel disease and for 2-vessel disease that includes the proximal LAD artery, particularly in individuals with diabetes and/or reduced LVEF [1-4]. These recommendations were made due to lower rates of repeat revascularization and lower mortality following CABG as compared with $\mathrm{PCl}$ in these patient populations.
However, with improvement in equipment, interventional techniques and drug therapy, $\mathrm{PCI}$ has gained momentum, being a less invasive procedure than CABG [2]. The benefit of CABG over PCl increases as the complexity of coronary artery disease worsens. In the setting of a low complexity coronary angiogram (SYNTAX score < 22), PCI is often considered as a viable alternative to CABG [2].

FFR is highly useful in coronary lesions with unclear significance on coronary angiography by providing a physiological assessment of hemodynamic severity. It is a valuable tool in clinical practice, especially in patients 

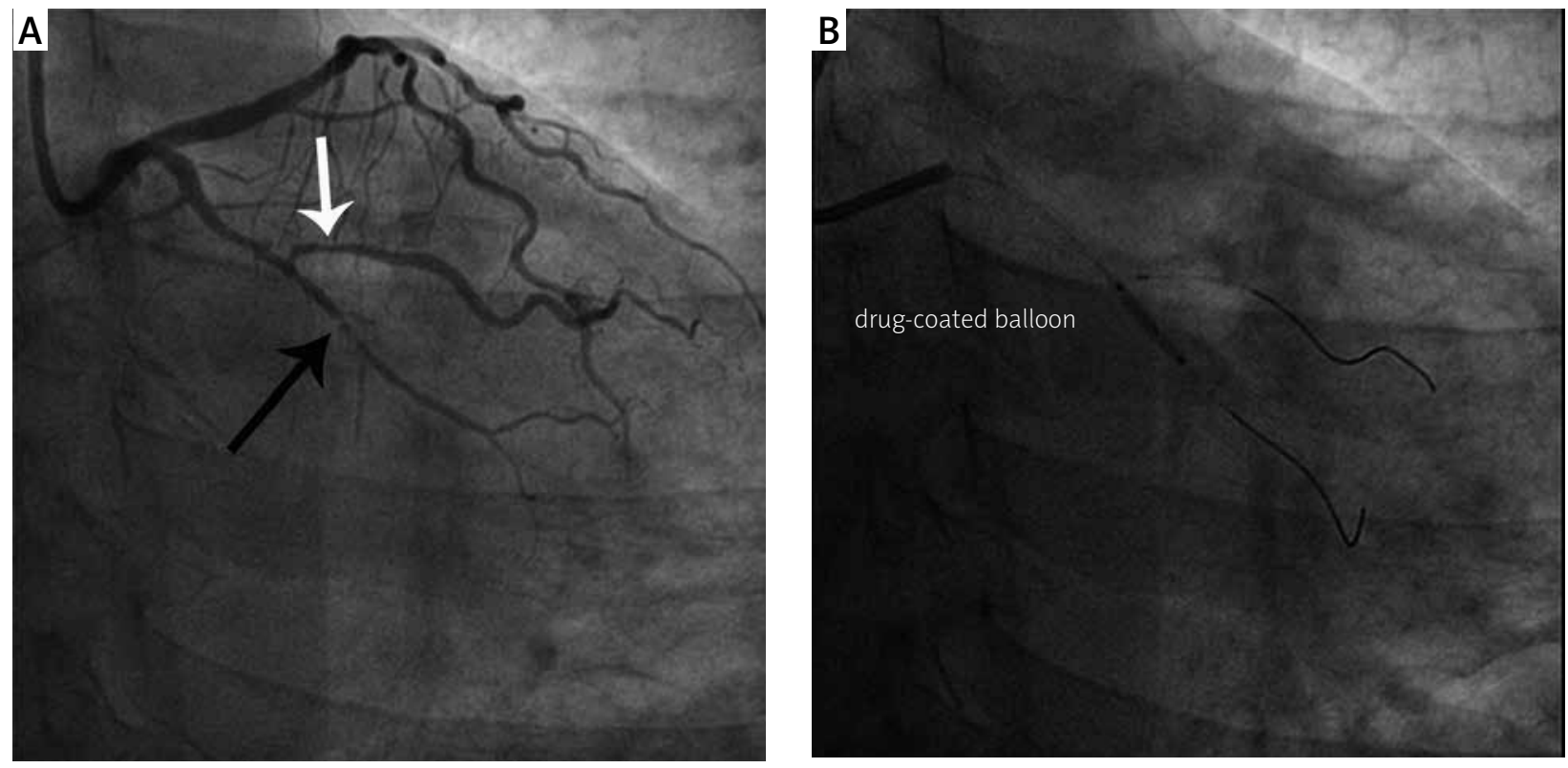

drug-coated balloon

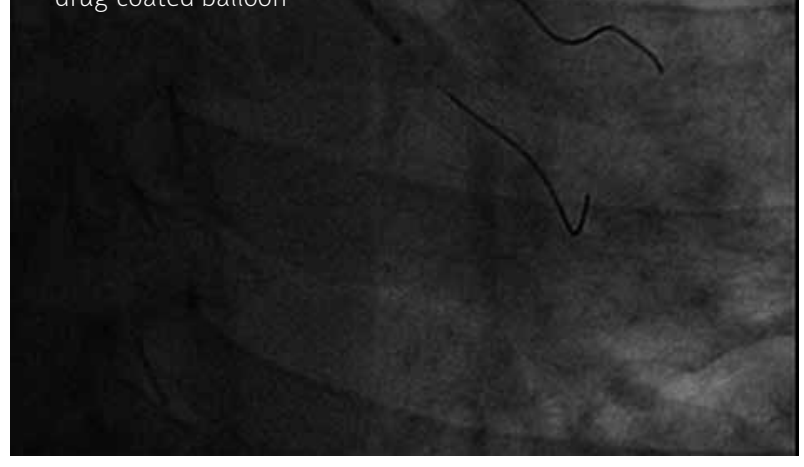

C

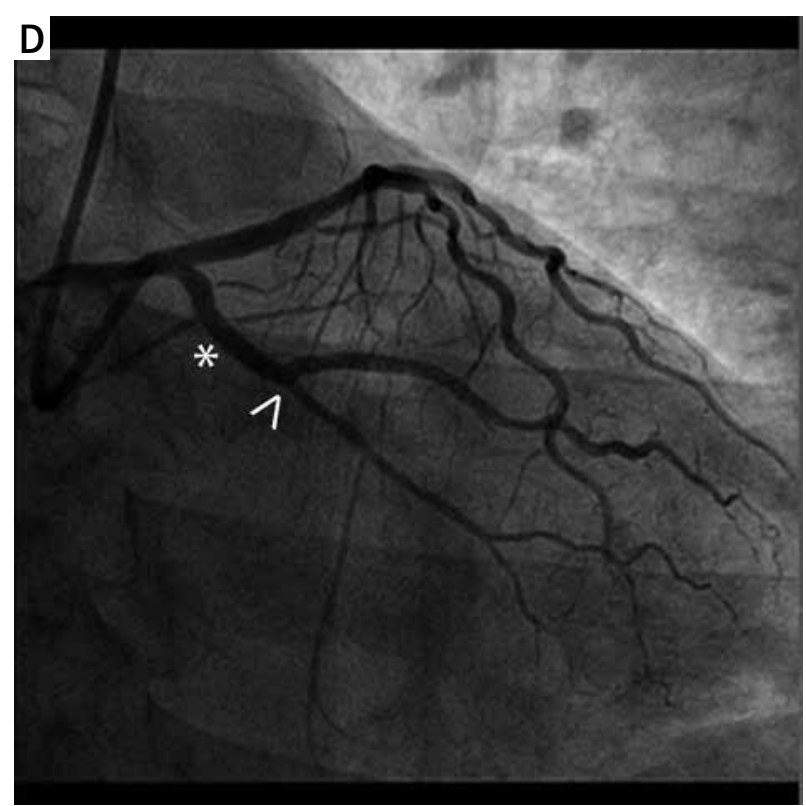

Figure 2. A - Angiography of the left circumflex (LCX) artery showing severe lesion in the proximal and mid part (black arrows) and severe ostial disease in the first obtuse marginal (OM1) branch (white arrow). B - Drug-coated balloon inflation to the mid LCX distal to the OM1 at $7 \mathrm{~atm}$. C - DES deployment in the proximal LCX to the OM1. D - Angiography showing a good angiographic result of the proximal LCX $\left(^{\star}\right)$ at the bifurcation with $\mathrm{OM} 1$ branch $\left({ }^{\wedge}\right)$ with no residual stenosis or dissection

with multivessel disease, since it allows one to avoid unnecessary treatment of hemodynamically insignificant lesions [5-7].

It is well accepted that coronary angiography does not allow visualization of the atherosclerotic burden within the arterial wall. In contrast, IVUS allows a real-time assessment of lumen area and plaque composition, size and distribution and permits evaluation of the appropriateness of stent expansion [8]. OCT, a light-based modality of intravascular imaging, has a higher spatial resolution than IVUS, thus enabling more accurate detection of intraluminal structures and subtle abnormalities. These include poor apposition of stent struts, residual thrombus, plaque prolapse and dissection. OCT-guided stenting may also improve clinical outcome [9]. Drug-coated balloons can be considered as alternatives to paclitaxel-eluting stents for the treatment of lesions in small caliber coronary arteries [10], and aiming to avoid complex bifurcation $\mathrm{PCl}$ using 2 stents, which is usually associated with higher rates of restenosis, a second stent 

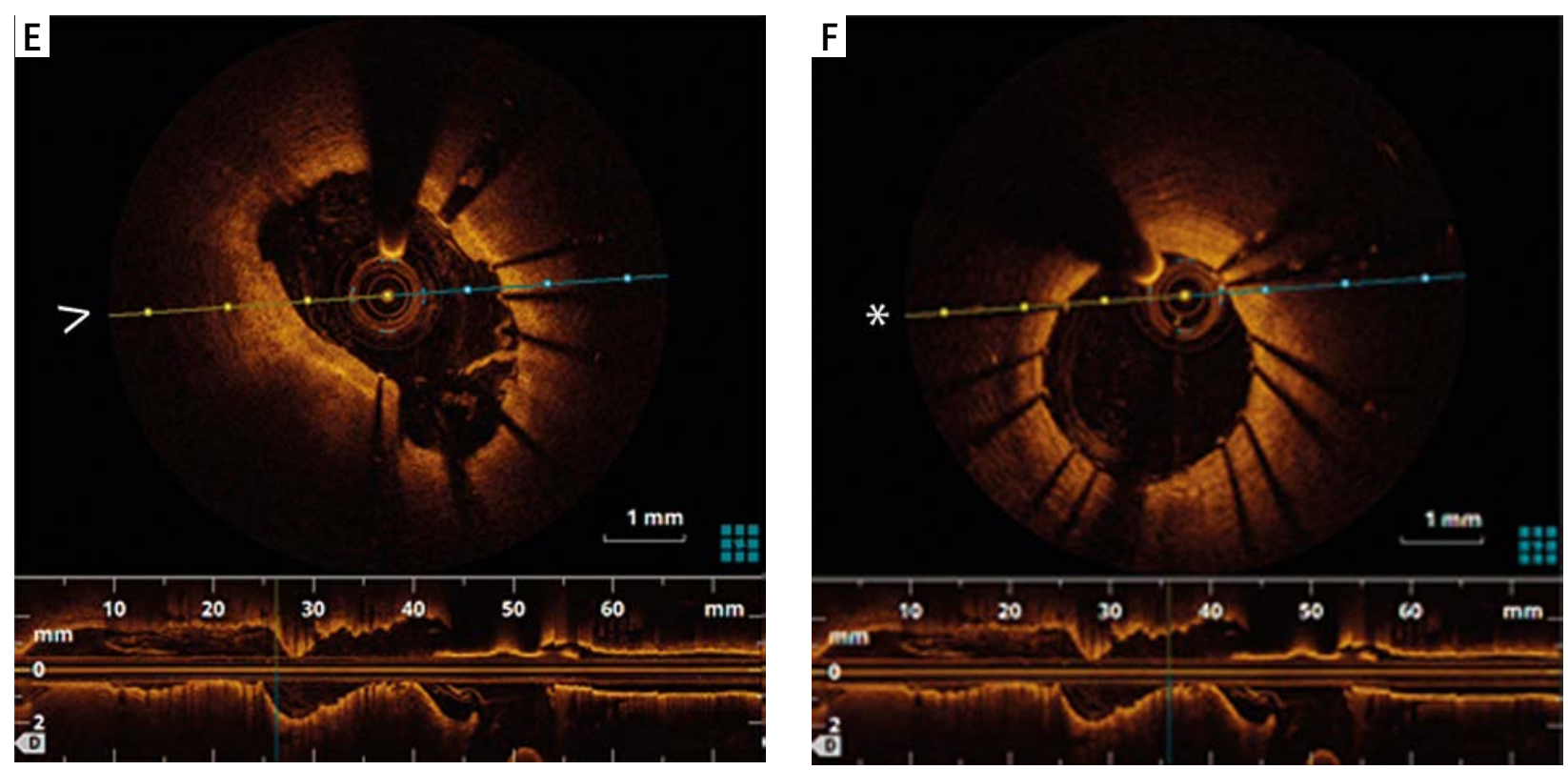

Figure 2. E, F - Post stenting optical coherence tomography (OCT) showing good strut apposition to the vessel wall in the mid part of LCX stent at the level of the bifurcation with the OM1 branch (E) and in the proximal part of the LCX (F)

was not implanted in the small caliber mid LCX and only a drug-coated balloon was used. The patient under discussion was fully covered by the social security service in Saudi Arabia. Nevertheless, we agree that physicians and healthcare deliverers should be conscious of the cost and benefits of procedures they perform. We aim at providing the most appropriate therapies to our patients, and we hope that our diagnostic and therapeutic decisions were cost effective in this particular patient.

\section{Conclusions}

In the present case, various interventional tools were utilized to optimize patient management. In the first procedure, FFR served to re-categorize the case from a severe 3-vessel to a 2-vessel disease that does not include the LAD, thus making $\mathrm{PCI}$ the preferred approach over CABG. Later IVUS was essential in understanding the mechanism of the proximal RCA lesion since it allowed identification of the presence of plaque dissection and later demonstrated optimal strut apposition of the stent to the vessel wall. Finally, a drug-coated balloon was used to treat the small caliber mid LCx artery and OCT was useful in confirming good strut apposition of the stent. Indeed, OCT was also appropriate to identify coronary dissection in the proximal RCA and to highlight apposition of struts after PCI, IVUS was used as per operator discretion. As demonstrated above, the utilization of modern interventional techniques allows for better understanding of the mechanism of individual lesions, helps assess hemodynamic severity of intermediate stenosis, aids in guiding stent deployment, and assists in clinical decision making, all of which may lead to improved patient outcomes.

\section{Conflict of interest}

The authors declare no conflict of interest.

\section{References}

1. Task Force on Myocardial Revascularization of the European Society of Cardiology (ESC) and the European Association for Cardio-Thoracic Surgery (EACTS); European Association for Percutaneous Cardiovascular Interventions (EAPCI), Wijns W, Kolh P, Danchin N, et al. Guidelines on myocardial revascularization. Eur Heart J 2010; 31: 2501-55.

2. Authors/Task Force members, Windecker S, Kolh P, Alfonso F, et al.; Authors/Task Force members. 2014 ESC/EACTS Guidelines on myocardial revascularization: The Task Force on Myocardial Revascularization of the European Society of Cardiology (ESC) and the European Association for Cardio-Thoracic Surgery (EACTS) Developed with the special contribution of the European Association of Percutaneous Cardiovascular Interventions (EAPCI). Eur Heart J 2014; 35: 2541-619.

3. Hlatky MA, Boothroyd DB, Bravata DM, et al. Coronary artery bypass surgery compared with percutaneous coronary interventions for multivessel disease: a collaborative analysis of individual patient data from ten randomised trials. Lancet 2009; 373: 1190-7.

4. Farkouh ME, Domanski M, Sleeper LA, et al.; FREEDOM Trial Investigators. Strategies for multivessel revascularization in patients with diabetes. N Engl J Med 2012; 367: 2375-84.

5. Pijls NH, van Schaardenburgh P, Manoharan G, et al. Percutaneous coronary intervention of functionally nonsignificant stenosis: 5-year follow-up of the DEFER Study. J Am Coll Cardiol 2007; 49: 2105-11. 
6. Tonino PA, De Bruyne B, Pijls NH, et al.; Investigators FS. Fractional flow reserve vs. angiography for guiding percutaneous coronary intervention. N Engl J Med 2009; 360: 213-24.

7. De Bruyne B, Pijls NH, Kalesan B, et al.; FAME 2 Trial Investigators. Fractional flow reserve-guided $\mathrm{PCl}$ versus medical therapy in stable coronary disease. N Engl J Med 2012; 367: 991-1001.

8. Zhang Y, Farooq V, Garcia-Garcia HM, et al. Comparison of intravascular ultrasound vs. angiography guided drug-eluting stent implantation: a meta-analysis of one randomised trial and ten observational studies involving 19,619 patients. Eurolntervention 2012; 8: 855-65.

9. Prati F, Di Vito L, Biondi-Zoccai G, et al. Angiography alone vs. angiography plus optical coherence tomography to guide decision-making during percutaneous coronary intervention: the Centro per la Lotta contro l'Infarto-Optimisation of Percutaneous Coronary Intervention (CLI-OPCI) study. Eurolntervention 2012; 8: 823-9.

10. Latib A, Colombo A, Castriota F, et al. A randomized multicenter study comparing a paclitaxel drug-eluting balloon with a paclitaxel-eluting stent in small coronary vessels: the BELLO (BalIoon Elution and Late Loss Optimization) study. J Am Coll Cardiol 2012; 60: 2473-80. 